bouteilles immédiate après la traite et appliquer un certain nombre de prescriptions d'hygiène générale, que l'on peut puiser dans les idées que je vous ai déjà exposées.

L'évolution vers un idéal meilleur est non seulement une question de morale, mais d'intérêt immédiat pour le producteur et le consommateur. Si nous laissons de côté la question d'intérêt, qui a sans doute une grosse importance pour notre économie nationale, pour ne retenir que la question morale, nous nous souviendrons de l'exemple de Straśbourg et nous penserons qu'il meurt en France une trop grande proportion de jeunes enfants parce qu'ils sont mal nourris.

Il ne faut plus que les Pouvoirs publics s'intéressent seulement au prix du lait, il faut aussi qu'ils viennent hâtivement au secours de la production pour l'aider à modifier ses méthodes car, on le sait, l'hygiène coûte cher.

Le lait, par conséquent, ne doit pas d'abord être bon marché ; il doit d'abord être bon. Un lait mal soigné est, même bon marché, trop cher ; un lait qui fait vivre est, même plus cher, bon marehé. Il ne faut plus que la question du lait se résume : pour le consommateur et pour les Pouvoirs publics, à une affaire de prix; pour le producteur, à une occasion de tracas sans grands profits.

II ne faut plus que les conditions économiques obligent le producteur à considérer le lait comme un sous-produit de la ferme. Que tous sachent bien qu'il constitue le sang de notre jeunesse, la vigueur de notre nation et que chacun augmente sa consommation pour vivre plus sainement, plus économiquement, en encourageant ainsi l'agriculture française à produire toujours meilleur.

\title{
REVUE
}

\section{UNE USINE MODERNE AMÉRICAINE POUR LA FABRICATION DE L'ACIDE LACTIQUE}

\author{
par \\ G. GENIN \\ Ingénieur E. P. C.
}

C'est à Hobart, dans l'Etat de New-York, qu'a été mise en route récemment la première usine américaine pour la fabrication de l'acide lactique et des lactates en partant du lactose. Cette usine appartient à la "Sheffield By-Produets Company " qui est une filiale de la "National Dairy Products Corporation" de New-York. La nouvelle usine est placée sous la direction de M. F. L. CHappel. 


\section{Utilisation du surplus de sérum.}

Cette Société avait été créée, il y a quelques années, dans le but de fabriquer de la caséine, du lactose brut et raffiné et de l'albumine. $\mathrm{Ce}$ n'est toutefois que depuis quelques semaines seulement qu'elle a pu travailler au maximum de sa capacité et utiliser le surplus du sérum dont elle disposait pour la fabrication des diverses qualités d'acide lactique et des lactates de sodium et de calcium. La capacité de production des nouveaux ateliers est estimée à environ 10.000 pounds par jour d'acide lactique à $22 \%$ ou son équivalent en dérivés de l'acide lactique. Il a d'ailleurs été prévu que cette production pourrait être doublée lorsque la consommation sera devenue suffisante.

L'acide lactique et les lactates fabriqués dans cette nouvelle usine sont au moins égaux, sinon supérieurs par leurs qualités, aux produits fabriqués par d'autres procédés. L'acide lactique par exemple obtenu dans cette usine se présente sous la forme d'un produit absolument incolore et complètement exempt d'impuretés, de sorte qu'il ne possède même pas l'odeur caractéristique de l'acide lactique ordinaire qui doit cette odeur à certains corps étrangers.

\section{Origine du procédé employé dans l'usine.}

Le procédé utilisé dans cette usine découle des travaux entrepris il y a quelques années par le Bureau de l'Industrie laitière du Ministère de l'Agriculture américain. Sous la direction du $\mathrm{D}^{\mathrm{r}} \mathrm{L}$. A. RoGERS, les Laboratoires du Bureau avaient étudié la possibilité de produire d'une façon continue du lactate de calcium en partant du sérum.

Les expériences avaient déjà montré que l'on peut, par un proeédé de fermentation, obtenir de l'acide lactique; on utilise comme milieu nutritif le sérum formé au cours de la fabrication de la caséine et comme organismes un mélange de Lactobacillus et de Mycoderm. Toutefois, on avait constaté, c'est d'ailleurs une caractéristique de toutes les réactions de fermentation, qu'un état d'équilibre était rapidement atteint pour une encentration relativement faible d'acide lactique et à partir de ce moment, les bactéries perdaient leur activité. C'est alors que, pour empêcher cet état d'équilibre de s'établir, le Bureau constata que l'on pouvait faire des additions successives de chaux hydratée ou de carbonate de calcium au milieu et transformer ainsi l'acide lactique en lactate de calcium. Toutefois, lorsque l'usine Sheffield entreprit la mise au point industrielle du procédé, on constata que la fabrication discontinue était plus avantageuse qu'une fabrication continue.

Le lactobacille employé qui est le $L$. bulgaricus additionné de mycoderm est préparé au Laboratoire en utilisant comme milieu 
nutritif du lait écrémé pasteurisé, dans lequel on assure la croissance des ferments à une température de $45^{\circ}$ environ. Après avoir été préparée dans des flacons de laboratoire, la culture est ajoutée au petit-lait pasteurisé contenu dans des boîtes d'une quarantaine de litres et c'est le lait ainsi inoculé qui est à son tour utilisé pour l'ensemencement du sérum stérilisé contenu dans des récipients en acier vitrifié d'une capacité de plus de 3.000 litres. Finalement on ajoute le contenu de 2 de ces récipients vitrifiés à une quantité suffisante de sérum pour obtenir un volume total d'environ 20.000 litres. Ce mélange est placé dans des cuves en bois d'une capacité d'environ $30 \mathrm{~m}^{3}$ où se fait la fermentation finale, le liquide étant légèrement agité. La température de $45^{\circ}$ est maintenue lorsque la croissance du ferment s'effectue, en faisant passer un courant de vapeur dans des serpentins immergés dans le liquide. Une durée de 48 heures est nécessaire pour que la fermentation d'une cuve soit terminée.

\section{Séparation du lactate de calcium.}

Lorsque la fermentation a commencé depuis 8 heures, on ajoute une certaine quantité de chaux éteinte sous forme de boue ; 4 additions successives de chaux sont faites au cours d'une préparation complète, de façon à ce que la eoncentration en acide lactique n'augmente jamais au delà de $1,3 \%$. Il faut pour chaque cuve de fermentation 900 à 1.000 pounds de chaux, un léger excès de chaux étant ajouté à la fin de la fermentation pour transformer la totalité de l'acide lactique en lactate de calcium.

Le liquide est alors porté à la température d'environ $90^{\circ}$ pour coaguler la lactalbumine, ce qui permet de séparer ce produit par sédimentation et décantation du liquide surnageant. On sépare donc le liquide que l'on recueille dans un récipient approprié et les sédiments, sous forme de boue, sont passés dans un filtre-presse, le filtrat étant ajouté au liquide. Ce liquide est traité avec du charbon activé ; on sépare celui-ci par filtration et la solution décolorée qui contient encore des sels inorganiques contenus dans le lait est dirigée dans un appareil d'évaporation à double effet où il est concentré jusqu'à $10^{\circ}$ Bé.

L'évaporateur que l'on utilise dans l'usine américaine est un appareil très moderne à grand débit qui comporte après chaque effet un dispositif pour la séparation de la vapeur. L'évaporateur à double effet est d'ailleurs complété de deux évaporateurs à simple effet. Lorsque l'on désire produire du lactate de ealcium, la solution est concentrée dans un des simples effets à $15^{\circ}$ Bé. Si l'on désire au contraire acidifier cette solution pour obtenir de l'acide lactique, il faut pousser sa concentration jusqu'à $20^{\circ}$ Bé, afin d'obtenir de l'acide lactique à $22 \%$. 
Voici comment on opère pour obtenir du lactate de calcium : la solution, concentrée à $15^{\circ}$, est dirigée par pompe dans un certain nombre de euves de eristallisation, refroidies par l'eau et disposées sur une galerie au-dessus des évaporateurs. L'usine dispose de 6 de ces cuves, chacune à double enveloppe, le récipient intérieur étant en acier stainless d'une capacité d'environ 1.200 litres. Des chicanes placées dans la double enveloppe assurent une circulation efficace de l'eau de refroidissement.

Lorsque les cristaux de lactate de calcium se sont séparés, les eaux-mères retournent dans les euves de fermentation, ainsi que les eaux de lavage des cristaux ; ces derniers sont séchés et ils sont purifiés s'il y a lieu pour être transformés en lactate de calcium répondant aux spécifications de la pharmacopée américaine.

Cette purification s'effectue comme suit : le lactate de calcium brut est dissous dans l'eau et la solution décolorée par une nouvelle addition de charbon actif. On procède à une nouvelle filtration, puis à une cristallisation et un lavage des cristaux. Ces cristaux sont séchés dans un sécheur tunnel pareouru par un courant d'air filtré, maintenu à une température rigoureusement contrôlée.

\section{Préparation de l'acide lactique.}

La solution de lactate de calcium qui doit servir à la préparation de 1'acide lactique est, rappelons-le, concentrée à $20^{\circ}$ Bé, aussi bien pour la fabrication de l'acide lactique industriel que pour la fabrication de l'acide lactique à $44 \%$ destiné à des usages alimentaires. Quant à l'acide lactique à $50 \%$ incolore et destiné également à ses applications alimentaires, il est préparé en partant du lactate de calcium de la pharmacopée.

Dans la fabrication de l'acide lactique industriel à $22 \%$, la solution concentrée de lactate de calcium est placée dans un bac de préeipitation où on ajoute de l'acide sulfurique à $66^{\circ}$ Bé pour transformer le lactate en acide lactique, avec précipitation de sulfate de calcium. La filtration du sulfate s'effectue dans un filtre-presse muni de plateaux et de cadres en ébonite et le filtrat est dirigé dans un réservoir où il est additionné de charbon actif, décoloré, puis finalement séparé du charbon dans un filtre à vide en grès. Le produit est alors prêt à être expédié.

Pour la fabrication de l'acide lactique plus concentré, on fait appel à une technique légèrement différente. C'est ainsi que pour la fabrication de l'acide à $44 \%$, on dissout le lactate de calcium industriel dans l'eau, et on ajoute l'acide sulfurique pour libérer l'acide lactique. On sépare le sulfate de calcium par filtration, et on ajoute au liquide une nouvelle quantité de lactate de calcium. On transforme à nouveau ce lactate de calcium en acide lactique et on parvient ainsi à obtenir une solution à 44 ou $50 \%$ d'acide lactique. 
Cette solution est finalement purifiée et décolorée au moyen du charbon actif, puis filtrée comme il a été dit plus haut.

On prépare le lactate de sodium technique ou purifié en faisant réagir l'acide lactique correspondant avec de la soude caustique. Le produit est concentré, mais est toujours vendu sous forme de solution.

\section{BIBLIOGRAPHIE ANALYTIQUE}

\section{LES LIVRES}

\section{JACQUot (R.) et NATAF (Berthe). - Le Manioc et son utilisation}

alimentaire. Actualités scientifiques et industrielles $n^{0} 364$, Nutrition. Préface du Professeur Emile F. Terroine. Edité par Hermann et Cle, 6, rue de la Sorbonne, à Paris, VIe. Nombreux tableaux et graphiques, volume de 56 pages. Prix 12 frs. 1936.

Nous sommes heureux d'attirer l'attention de nos lecteurs sur la série de Monographies groupées sous le titre général "Nutrition ", exposés publiés sous la direction de M. le professeur TERROINE, directeur de l'Institut de Physiologie générale à la Faculté des Sciences de Strasbourg. Cette collection a pour but essentiel de lutter contre les méfaits d'une spécialisation outrancière et de permettre aux différents techniciens s'oecupant de la même question, mais dans un esprit quelquefois différent, de ne pas s'ignorer les uns les autres.

"Le manioc et son utilisation alimentaire p porte le $n^{0} 1$ de cette collection.

Dans cette monographie, R. J. et B. N. ont donné d'abord les earactéristiques botaniques et chimiques du manioc (description botanique, culture, variétés, composition chimique : amidon, substances azotées, substances minérales, vitamines). Le deuxième chapitre traite de la physiologie alimentaire du manioc (digestibilité, valeur énergétique, valeur amidon, le manioc est-il un aliment complet ?).

L'emploi et les avantages nutritifs du manioc sont exposés largement dans un chapitre d'application qui traite de l'engraissement des porcs, de l'allaitement et de l'engraissement des veaux, des différents usages, de la meunerie, ot de la panification. Enfin le dernier ehapitre intéresse l'économie du manioc (supériorité économique, rendement, exportation, importation).

Un examen impartial montre, d'après cet exposé, que les avantages présentés par le manioc sont bien supérieurs à ses inconvénients. Les inconvénients de ce féculent présentent souvent des remèdes faciles. Il existe des maniocs doux, comme celui de Madagasear, dépourvus d'acide cyanhydrique ; et, d'autre part, la preuve de l'inocuité, à l'aide du papier picro-sodé, est très facilement réalisable. La déficience du manioc en protéine peut être aisément comblée par la viande ou le sang desséché. Une faible dose de phosphate compensera la pauvreté du manioc en phosphore. Le manioc présente, avec 